\title{
Relational Nouns and Reciprocal Plurality
}

\author{
Peter Staroverov \\ Moscow State University
}

\section{Introduction}

This paper investigates the behavior of relational nouns (hereafter RNs) in conjoined and plural contexts. Relational nouns can be roughly defined as nouns having more than one argument (DeBruin and Scha 1988, Lander 2000). Sometimes it is difficult to say if a noun belongs to this class or not (cf. boss, picture). A noun may have both a relational and a non-relational (sortal) reading (cf. Vikner and Jensen 2002: 204-205). However, all the examples I give in this paper are clear examples of RNs. Throughout this paper, I will follow Lander (2000) in using the terms referent and correlate to refer to the two arguments of RNs.

Semantic analysis of plurality and coordination as applied to nominal categories so far has mainly focused on one-place nouns. In this paper, I will argue that generalizing the proposed semantic mechanisms to RNs is not trivial. The problematic case of coordination is illustrated in (1).

(1) The novel is about a husband and wife

The two conjoined RNs in (1) refer to two people who are husband and wife of each other and cannot refer to, say, speaker's husband and listener's wife. Such reciprocal conjunction is not easy to capture within the existing theories of conjunction. For instance, the account of Winter (2001) predicts that both arguments of the conjoined semantic predicates should always end up coreferent. This is clearly not the case in (1) where the referent of the first RN is coreferent to the correlate of the second one and vice versa. In Section 3, I will demonstrate that the recently proposed alternatives to Winter (2001), e.g. Heycock and Zamparelli (2005), are generally hard to extend to the case of two-place noun coordination.

For the plurals, an analogous interpretation arises in examples like brothers meaning 'brothers of each others'. I will argue that apparent similarity in the meanings of the two classes of examples is not accidental. I will refer to both cases as the instantiations of reciprocal plurality. I propose an analysis that derives the two interpretations in a similar way and that fits well with lexical restrictions on reciprocal plurality.

This work was supported by the Russian Foundation for Basic Research (grant 07-06-080203 to Peter Staroverov). I would like to thank Barbara Partee for encouraging me to carry out this work and for her extremely useful comments. At different stages of this research, I benefited from discussion with Igor Yanovich, Eytan Zweig, James Pustejovsky, Laurence Horn and Mary Dalrymple. I would like to thank the audiences at Nova Gorica, Moscow and Storrs, in particular Philippe Schlenker, Ora Matushanski, Ken Shan, Pranav Anand and Chris Barker. 
The rest of the paper is laid out as follows: in Section 2, I compare the coordination of sortal nouns and RNs. I present an analysis of reciprocal coordination in Section 3. Section 4 is devoted to reciprocal plurals and I discuss lexical restrictions on reciprocal plurality in Section 5. Sections 6 and 7 conclude the paper.

\section{Relational Nouns Conjunction versus Sortal Nouns Conjunction}

In this Section, I will argue that there are at least two types of interpretation available for sortal nouns in coordination construction. These two interpretations are also available for RNs. However, there is one more interpretation that is only available to certain RNs and not available to any sortal nouns. As such, this interpretation cannot be treated either as a case of intersective conjunction or as a case of group-forming conjunction.

\subsection{Intersective Conjunction}

This interpretation of nominal coordination was first proposed as part of a broadly cross-categorial generalization of boolean (sentential) conjunction. Other terms used in the literature for the same interpretation include generalized conjunction (Partee and Rooth 1983), boolean and (Krifka 1990), joint reading (Heycock and Zamparelli 2005).

For the case of $<\mathrm{e}, \mathrm{t}>$-type expressions this interpretation amounts to set intersection.

\section{(2) John is a liar and thief}

For instance, in (2) both conjoined properties apply to the same individual. Intersective conjunction schema also derives the right result for DP-conjunction. Winter (2001) proposes a way of generalizing this schema to some cases of collectivity.

What is important for our current purposes is that intersective conjunction can also occur with RNs as illustrated in (3).

(3) Alexander is my friend and colleague

In this example, the conjoined RNs have both the same referent and the same correlate. If we assume that the denotations of RNs are sets of pairs, we immediately get the right interpretation by intersecting the set of pairs $\langle x, y\rangle$ such that $x$ is a friend of $y$ with the set of pairs $\langle u, v>$ such that $u$ is a colleague of $v$.

\subsection{Group-forming Conjunction}

The intersective conjunction schema cannot capture the examples like (4) where the whole conjoined phrase takes just one determiner. In the literature, the terms 
split reading (Heycock and Zamparelli 2005), and non-boolean conjunction (Krifka 1990) have also been used to refer to such examples.

This man and woman are in love ${ }^{1}$

Roughly speaking, the problem here is that man and woman does not refer to one entity that is both a man and a woman at the same time. The variables in the denotations of conjoined nominal expressions are not bound and hence the intersective schema imposes identity on the two referents.

Heycock and Zamparelli (2005) attempt to propose a unified meaning for and based on the cases like (4) ${ }^{2}$. However, they give only a very tentative idea of how their account can be generalized to cover the intersective conjunction in case of DP coordination and coordination of other categories.

Furthermore and more importantly, there is no simple way to generalize their account to two-place nouns. The semantic operation that they assume to correspond to coordination (set product) essentially picks out all the members of the set denotations of the conjuncts and returns a set containing the unions of those members in all possible combinations.

$$
S P\left(S^{1}, \ldots, S^{n}\right)=\left\{X: X=A^{1} \cup \ldots \cup A^{n}, A^{1} \in S^{1}, \ldots, A^{n} \in S^{n}\right\}
$$

If we assume that RNs denote sets of pairs (not sets of individuals as Heycock and Zamparelli assume), the denotation of a phrase like friend and colleague should contain, among others, a set of two pairs $\{\langle\mathrm{x}, \mathrm{y}\rangle,\langle\mathrm{u}, \mathrm{v}\rangle\}$ where $x$ is a friend of $y$ and $u$ is a colleague of $v$. However, there is no context in which friend and colleague can refer to a friend of $y$ and a colleague of $v$ with all the four individuals distinct. It is not clear what might be the mechanism that would filter out the undesired pairs from the denotation of coordinate structure.

Note that the assumption that RNs denote sets of pairs is justified by numerous works on the semantic, pragmatic and morphological behavior of RNs (see Asudeh 2005, Lander 2000, Vikner and Jensen 2002, De Bruin and Scha 1988, Partee 1989, Barker 1999 among others).

The only account that was explicitly generalized to two-place nouns to my knowledge is the more conservative Linkean account assuming that and is in some cases used to form groups out of individuals. Krifka (1990) generalizes Link's $\oplus$ operator to apply to arbitrary types. As $\oplus$ forms groups, this analysis immediately predicts that the whole conjunction quantifies over groups and hence can take just one article.

For the case of RNs, Krifka's generalization of group formation predicts that two conjoined nouns should have different referents but the same correlate.

\footnotetext{
${ }^{1}$ Barbara Partee (p.c.) notes that the acceptability of such examples may be questionable. This particular example is taken from Heycock and Zamparelli (2005). I accept their judgements but further research might be needed to find more well-established examples of this kind.

${ }^{2}$ Winter (2001) tries to generalize the intersective schema to all cases by stipulating wide scope for conjunction in the cases like every cat and dog. I agree with the criticism of Winter's approach in Heycock and Zamparelli (2005).
} 
This is indeed a plausible interpretation for conjoined RNs combined with possessors (6).

John's girlfriend and brother came to his party

On the group-forming analysis, this example should roughly mean that a group of people that has two subparts came. The first subpart was John's girlfriend and the second one - his brother.

Several alternatives to Krifka's account have been proposed (see the references in Winter 2001, chapter 2). I do not aim to make a motivated choice between those here, as my main concern is the reciprocal interpretation. What is important however is that the group-forming approach captures the occurrences of conjoined RNs with possessors.

\subsection{Reciprocal Conjunction}

The reciprocal conjunction is illustrated in (1), repeated here as (7).

The novel is about a husband and wife

Interestingly, the reciprocal conjunction is the only type of conjunction interpretation that cannot occur with sortal nouns. The very basic semantic properties of reciprocal conjunction require the conjuncts to have two arguments. In what follows, I will consider the relation between the reciprocal conjunction and other types of conjunction briefly described in the previous sections.

First of all, the reciprocal conjunction cannot be captured by the intersective schema because the intersection of the noun denotations in question is often empty. Consider, for example, the pair brother and sister. The set of pairs $<x, y>$ such that $x$ is a brother of $y$ clearly does not intersect with the set of pairs $<u, v>$ such that $u$ is a sister of $v$. The first members of each pair in the first set are males but the first members of each pair in the second set are females. In other words, one person cannot be both a sister and a brother to some other person. Therefore, the intersective schema cannot be applied in this case. Similar reasoning is valid for husband and wife, teacher and pupil and many other examples of reciprocal conjunction.

In what follows, I will demonstrate that any account of group-forming conjunction cannot capture the reciprocal reading either. This becomes clear as we notice that the group-forming conjunction can apply to RNs in question to produce examples ambiguous between the reciprocal and the group reading. These examples come from conjoined RNs in argument positions.

John invited an uncle and nephew to the party

$(8)^{3}$ is clearly ambiguous between the reciprocal reading on which uncle and nephew are related to each other but are not John's relatives and the group reading on which they are John's uncle and nephew (and hence probably a great-

\footnotetext{
${ }^{3}$ Special thanks are due to Barbara Partee for pointing the English examples of this kind to me.
} 
uncle and great-nephew of each other). I suggest that the reciprocal reading of (8) is derived by the same mechanism as the reciprocal reading in (7), while the nonreciprocal reading occurs as a result of group-forming conjunction. As the example (8) is clearly ambiguous we obviously need to distinguish between two different interpretations here.

To sum up, the existing approaches to coordination semantics cannot capture the reciprocal conjunction. This makes us face the question about the ambiguity of and. Is the reciprocal interpretation yet another meaning derived by specific mechanisms? The reciprocally conjoined phrases seem to share some properties with group-forming conjunction and some other ones - with intersective conjunction.

On the one hand, most of the RNs giving rise to reciprocal interpretation in coordination construction have disjoint denotations. For instance, the denotations of teacher and pupil, employer and employee, father and son have an empty intersection. This makes the distribution of intersective conjunction and reciprocal conjunction close to complementary. Hence, an analysis that would treat the reciprocal interpretation as a variant of the intersective one is a plausible one.

On the other hand, the conjoined phrases involving reciprocal conjunction allow for just one article ${ }^{4}$, as illustrated in (8). This suggests that the whole reciprocally conjoined phrase quantifies over groups. The next section provides a compositional analysis that accounts for those dual properties and derives the reciprocal conjunction without postulating an additional meaning of and. One further argument in favor of such an analysis is that we normally do not get examples that are 3-ways ambiguous between intersective, group-forming and reciprocal conjunction.

\section{Reciprocal Conjunction: Analysis}

I propose to derive the reciprocal interpretation in three essential steps. At the first stage, the denotations of RNs are adjusted to make the intersective schema applicable. Second, the intersective conjunction applies. The third stage is the application of a special collectivity operator responsible for group-like behavior of the reciprocally conjoined phrases.

\footnotetext{
${ }^{4}$ In fact even a more precise formulation seems to be true: the reciprocal conjunction requires just one article. For instance, the sentence in (7) could not get a continuation like "who were not married to each other". Consider on the contrary the amazon.com description of some movie:

(i) A hilarious movie about a husband and a wife who fall in love. Only they are not married to each other.

The reciprocity in this case becomes a pragmatic matter. As suggested to the author by Barbara Partee (p.c.), such cases in English can be derived by the intersective conjunction of DPs. In this case the relational nouns shift to one-place predicates by existentially quantifying the correlate in order to combine with the ordinary version of the article. The "relational" version of $a$ proposed in Partee (1999) would lead to a crash in derivation. To derive the "default" reciprocal meaning of $a$ husband and a wife we might appeal to a plausible pragmatic principle that would always require the conjuncts to be somehow related (first suggested to me by Igor Yanovich). We leave the detailed examination of such pragmatic possibilities for future research.
} 
The intersective conjunction schema (Winter 2001: 23) is defined as follows:

$$
{ }^{*}{ }_{\tau(\tau \tau)}=\left\{\begin{array}{lr}
\wedge_{t(t t)} & \text { if } \tau=\mathrm{t} \\
\lambda X_{\tau} \cdot \lambda Y_{\tau} \cdot \lambda Z_{\sigma_{1}} \cdot X(Z)^{*}{ }_{\sigma_{2}\left(\sigma_{2} \sigma_{2}\right)} Y(Z) & \text { if } \tau=\sigma_{1} \sigma_{2}
\end{array}\right.
$$

In Section 2, I have argued that this schema cannot be directly applied to two RNs like brother and sister because their denotations have an empty intersection. However, it is important to notice that this schema can be applied to such RNs and give a non-empty intersection if the arguments of one of the RNs get inversed. On my account, this inversion happens to the second RN. The operator responsible for the inversion is defined in a following way:

$$
i n v_{(e e t)(e e t)}=\lambda Y_{e e t} \cdot \lambda u \cdot \lambda v \cdot Y(v)(u)
$$

In most cases, this operator may be said to be triggered by the fact that RNs in question have an empty intersection. The application of inv automatically restricts the reciprocal conjunction to pairs of nouns and to nouns having just two arguments. This is the correct result, as we have no evidence of more-than-2place nouns giving rise to the reciprocal conjunction or of the conjunctions of more than two nouns licensing the reciprocal interpretation.

As noted above, after the application of inv the intersective schema can be applied to, say, $\operatorname{brother}^{\prime}(x)(y)$ and $\operatorname{sister}^{\prime}(y)(x)$. The successive application of the operators in (10) and (9) gives the result below.

$$
\lambda x . \lambda y\left[R_{1}(x)(y) \wedge R_{2}(y)(x)\right]
$$

This is then an input to a special collectivity operator that essentially takes a relation and returns a pair of individuals connected by that relation ${ }^{5}$.

$$
\lambda R \lambda Z \exists x \exists y[Z=x \oplus y \wedge R(x)(y)]
$$

The $\oplus$ in this formula can be viewed as a standard group-forming operator of Link (1983) ${ }^{6}$. The application of (12) correctly describes the quantificational properties of reciprocal conjunction. The resulting semantic representation of brother and sister is given in (13).

\footnotetext{
${ }^{5}$ As pointed out by Ken Shan (p.c.), we need to ensure that the operator in (12) does not apply to single RNs deriving the meaning of reciprocally conjoined phrases. To do this we might stipulate that the operator only applies to plurally specified relations $(\mathrm{say},[+\mathrm{pl}]$ in the sense of Heycock and Zamparelli 2003 and Roodenburg 2004). The question, however, bears on a more general issue of restrictions on type-shifting. In this paper I adopt the so-called lazy strategy (Winter 2001, Partee and Rooth 1983) that prohibits type-shifting if it's not needed.

${ }^{6}$ The choice of exact ontological status of groups is irrelevant for our current purposes. I take the approach of Schwarczhild (1992) and Krifka (1991) as it is easily compatible with ** operator (see Section 4).
} 


$$
\lambda Z \exists x \exists y\left[Z=x \oplus y \wedge \operatorname{brother}^{\prime}(x)(y) \wedge \operatorname{sister}^{\prime}(y)(x)\right]
$$

This formula can roughly be translated as "a pair of individuals $x$ and $y$ such that $x$ is a brother of $y$ and $y$ is a sister of $x$ ".

My analysis derives the right result when such pairs occur in predicative contexts, (14), given the standard assumptions about the semantics of copula.

\section{Roger and Susan are brother and sister}

The derivation proposed above includes the application of intersective conjunction schema. Hence, we treat the reciprocal conjunction as a variant of intersective conjunction. We do not postulate a third special meaning of and for reciprocal cases. Such an account successfully avoids postulating the redundant ambiguity as intersective conjunction and reciprocal conjunction are in most cases complementarily distributed.

A possible alternative to the 3-step derivation proposed above would be to design a single operator that applies to two relations and to integrate the inversion of arguments into this operator ${ }^{7}$. However, such an analysis would have to evoke additional speculations explaining why the 3-ways ambiguous examples are extremely rare (if at all possible, cf. Section 5).

In what follows, I will try to provide more motivation for the analysis described above. This motivation comes from two sources: first, this analysis is easily extendible to reciprocal plurals like brothers and second, it coincides quite well with the lexical restrictions on reciprocal interpretation. Section 4 deals with reciprocal plurals and Section 5 analyzes the lexical restrictions.

\section{Reciprocal Plurals}

In parallel to reciprocal conjunction, some plural RNs also give rise to reciprocal interpretation (Eschenbach 1993). Thus, sisters, friends, colleagues, neighbours can mean "sisters of each other", "friends of each other" etc.

The similarity between reciprocal plurals and reciprocal conjunction is not purely semantic. Both types of examples can occur in predicative position ${ }^{8}$.

a. John and Mary are husband and wife/spouses

\footnotetext{
${ }^{7}$ This idea was first suggested to me by Barbara Partee.

${ }^{8}$ Heycock and Zamparelli (2003) and Mary Dalrymple (p.c.) point out that the reciprocally conjoined and plural RNs often allow for no determiner in predicative position (15a) and in generic sentences. However, based on the examples like (i) they claim that this possibility is not limited to RNs and reciprocal conjunction (cf. also Roodenburg 2004).

(i) He was judge, jury and executioner

The availability of similar examples with group-forming conjunction and the integration of my analysis with the analysis of coordinate bare nominals remain matters of future research.
} 
Furthermore, as I will demonstrate in Section 5, the lexical restrictions on both types of interpretation are quite similar. The apparent similarity in the behavior of two interpretations makes it desirable to derive them both in a similar way. In what follows, I will demonstrate how my analysis of reciprocal conjunction can be extended to cover the cases of reciprocal plurals. I will refer to both cases as the instantiations of reciprocal plurality.

\subsection{Previous Proposals.}

In this section, I will briefly summarize and evaluate the previous analyses of reciprocal plurals. I will first consider the analysis of Hackl (2002) and then turn to Eschenbach (1993).

Martin Hackl suggests that the derivation of reciprocal plurals (and even of a broader class of essentially plural predicates) starts with insertion of a silent reflexive pronoun. Thus, the reflexivized version of next-door neighbour looks like $\lambda x$.next_door_neighbour' $(x)(x)$.

This clearly yields an empty set as it stands, which leads Hackl to assume that the plural RNs have to be cumulative. He suggests that the double star operator (Krifka 1986, Beck 2000) always applies after the reflexivization. The resulting interpretation next_door_neighbour ${ }^{* *}(x)(x)$ roughly refers to a set in which for every individual $x$ there is another individual $y$ such that next_door_neighbour' $(x)(y)$ and next_door_neighbour' $(y)(x)$.

This implies that for all cases of reciprocal plural RNs the cumulative inference patterns should be valid. For instance, if next_door_neighbour' is true of pairs $<$ John, Mary $>$ and $<$ Mary, Sue $>$ it should follow that John, Mary and Sue are next-door neighbours.

However, native speakers of English report that it is doubtful that those patterns are valid for all plural reciprocal RNs. To my mind, assuming that reciprocal plurals can only have weak interpretation leads to more serious conceptual problems as the cumulativity can be generalized infinitely. For instance, it implies that, if there is a neighbourhood chain, all the inhabitants of some city are predicted to be neighbours (Eschenbach 1993: 19). Furthermore, on Hackl's analysis, it is not clear why the reciprocal meaning should be connected with reflexive pronoun insertion. For instance, Barker (1999) argues that forming a reflexive predicate out of RNs is an unfavored operation that requires additional contextual support.

Finally, it remains unclear if the derivation proposed by Hackl is lexical or syntactic. In fact, Hackl (2002: 178-180) finds arguments in favor of both approaches.

To sum up, the approach of Hackl (2002) leaves some issues unresolved. In what follows, I will consider the analysis of Eschenbach (1993). To derive the reciprocal meaning of plural RNs Carola Eschenbach proposes a special rec operator that applies to the denotations of RNs. This operator derives a reciprocal plural meaning out of the singular meaning of a RN. The formal definition of ${ }^{\text {rec }}$ is given in (16) where I use a slightly more straightforward notation than Eschenbach's original one. $\subseteq$ and $\cap$ are used as generalized order and set intersection symbols. 


$$
{ }^{r e c}=\lambda R \lambda Z(\operatorname{cmplx}(Z) \wedge \forall x, y \subseteq Z[x \cap y=\varnothing \rightarrow R(x)(y)])
$$

Roughly speaking, this operator takes a relation as input and returns a set $Z$ of individuals such that each pair in the set is connected by the original relation (the fact that $\mathrm{Z}$ is a complex object is reflected by the presence of a predicate cmplx). Thus, for sister' the output will be as in (17).

$$
\lambda Z\left(\operatorname{cmplx}(Z) \wedge \forall x, y \subseteq Z\left[x \cap y=\varnothing \rightarrow \operatorname{sister}^{\prime}(x)(y)\right]\right)
$$

One important difference from Hackl's proposal is that on Eschenbach's account the reciprocal plurals quantify over groups and are not cumulative. In other words, Eschenbach endows the reciprocal plurals with a strongly reciprocal reading.

As in our analysis of coordination, the interpretation of plural RNs combined with possessors (sisters of Peter) is derived by completely different mechanisms than the reciprocal one. In fact, on Eschenbach's analysis this interpretation is a result of application of ct-pl operator that is responsible for count plurals (18).

$$
\boldsymbol{c t}-\mathbf{p l}=\lambda P \lambda x(\operatorname{cmplx}(x) \wedge P(x))
$$

Contrary to its application to sortal nouns, ct-pl combines with RNs by functional composition. The resulting meaning of sisters of Peter is $\lambda x\left(\operatorname{cmplx}(x) \wedge \operatorname{sister}^{\prime}(p)(x)\right)$.

The account of Eschenbach (1993) probably does better for the case of reciprocal plural RNs. However, the two derivations are not strictly speaking incompatible and hence we might expect to get a weak reciprocal reading derived along the lines of Hackl (2002) in some cases.

Both Hackl and Eschenbach attempt at formulating the adequate lexical restrictions on reciprocal plurality. The restrictions they formulate are similar, but not identical. Hackl (2002) claims that to give rise to a reciprocal plural reading a RN should denote a symmetric and irreflexive relation. This seems to be too loose, as, for instance, sister ${ }^{\prime}$ is not strictly speaking symmetric: $\operatorname{sister}^{\prime}(x)(y)$ does not imply $\operatorname{sister}^{\prime}(y)(x)$.

The lexical restriction proposed by Eschenbach (1993) is that the RN should be non-antisymmetric. This coincides well with her definition of ${ }^{\text {rec }}$ as this ensures the non-emptiness of the set $Z$ in (16). However, as I will argue in Section 5 , this restriction is not always satisfied. Before we turn to lexical restrictions however, I would like to describe the connection between my analysis of reciprocal conjunction and Eschenbach's analysis of reciprocal plurals in more detail.

\subsection{The Relation between my Analysis of Reciprocal Conjunction and Eschenbach's Proposal}

Notice, that, although Eschenbach's formula captures the desired interpretation, it does not state the reciprocity requirement straightforwardly. As the elements of 
the set $Z$ in (16) can be permutated, this formula is in fact equivalent to the requirement that $R$ holds of pairs $(\mathrm{x}, \mathrm{y})$ and $(\mathrm{y}, \mathrm{x})$ for every two elements belonging to the set. In other words, it is equivalent to (19).

$$
{ }^{r e c}=\lambda R \lambda Z(\operatorname{cmplx}(Z) \wedge \forall x, y \subseteq Z[x \cap y=\varnothing \rightarrow R(x)(y) \wedge \operatorname{inv}(R(x)(y))])
$$

Because (16) and (19) are truth-conditionally equivalent, it would be hard to argue between the two interpretations. One way or another, the availability of (19) indicates that different instantiations of reciprocal plurality can be analyzed in a similar fashion.

Such an analysis, I believe, should be grounded in the application of inv. In fact, this analysis allows us to treat the reciprocal plurals as a special kind of collectivity operator imposed on the output of inv and intersective conjunction applied to just one relation. This hypothetical input to collectivity is given in (20) below.

$$
\rho=\lambda x \lambda y(R(x)(y) \wedge \operatorname{inv}(R(x)(y)))
$$

Compared to the operator in (12), the collectivity operator that should apply to (20) differs in that it contains universal quantification instead of existential. One way or another, an analysis of reciprocal plurals involving the application of inv supports the initial idea of Krifka (1990) about the close semantic relationship between conjunction and plurals.

Finally, this analysis provides an account of the weakly reciprocal cases like (21) without any reference to covert reflexives.

In this city the professors and the students are neighbours

Intuitively, for this example to be true, it should not be the case that every professor is neighbour of every student. Rather, for every professor there should be a student such that they are neighbours. This is exactly the weak reciprocal interpretation derived by ** operator and predicted by Hackl's (2002) analysis.

It is not hard to see that this interpretation can be achieved by applying ** to the relation $\rho$ defined in (20). In fact, if the professor $\mathrm{p}_{1}$ is a neighbor of the student $\mathrm{s}_{1}$ and the same is true for $\mathrm{p}_{2}$ and $\mathrm{s}_{2}$ but no more neighbourhood relationships can be established in a given city, the relation $\rho^{* *}$ will be true as well as the sentence $(21)^{9}$.

Hence, my analysis preserves the initial idea of Hackl (2002) that reciprocally plural RNs can be weakly reciprocal but does not appeal to the insertion of a silent reflexive, which is arguably an undesired operation (Barker 1999).

To sum up, the application of inv allows me to extract a common part out of the semantic representations of reciprocal conjunction and reciprocal plurals. This common part is the expression in (20) (and (11) above). The differences in

\footnotetext{
${ }^{9}$ This raises the question of choosing between strong and weak interpretation in a given context. I believe, this choice depends on the presence of a contextual cover (cf. Beck 2000, Schwarzschild 1992 among others).
} 
semantics between reciprocal plurals and reciprocally conjoined phrases arise from two sources on this account. First, in conjoined phrases we deal with two relations while in plurals the same relation occupies both places. Second, the collectivity operators applied in the two cases are different: the conjunction operator (12) contains existential quantification while the plural operator universal. Finally, the weak reading of reciprocal plural RNs can be accounted for by applying double star to the same basic expression (20).

\subsection{Conjoined Plurals}

In this section, I will look at the predictions of my analysis for the case of conjoined plurals like brothers and sisters. I would like to start with the assumption that the relations denoted by RNs can hold not only between individuals but also between sets and between sets and individuals. This is needed to make inv applicable to the denotations of plural RNs like brothers.

The table below summarizes the predicted range of possible interpretations for brothers and sisters.

\begin{tabular}{|l|l|l|}
\hline Plural & Conjunction & Example \\
\hline reciprocal & $\begin{array}{l}\text { group- } \\
\text { forming }\end{array}$ & $\begin{array}{l}\text { a. Brothers and sisters create different kinds of } \\
\text { atmosphere in the family. }\end{array}$ \\
\hline $\begin{array}{l}\text { non- } \\
\text { reciprocal }\end{array}$ & reciprocal & $\begin{array}{l}\text { b. Brothers and sisters always prefer to live together } \\
\text { at least on one of the readings: brothers and their } \\
\text { sisters) }\end{array}$ \\
\hline $\begin{array}{l}\text { non- } \\
\text { reciprocal }\end{array}$ & $\begin{array}{l}\text { group- } \\
\text { forming }\end{array}$ & c. John's brothers and sisters came to his party. \\
\hline
\end{tabular}

Table 1: The interpretations of brothers and sisters

As we see, the range of possible interpretations of conjoined plural RNs is predicted by different combinations of the meanings of conjunction and plural morpheme. In addition to this picture, there are some examples that apparently arise due to the application of double star operator. Thus, the cumulative plural interpretation of RNs can combine with reciprocal conjunction to derive the meaning that roughly can be paraphrased as "the set of n-tuples such that for all the tuples the relations in question hold", cf. $(22)^{10}$.

(22) We want brothers and sisters for the roles of the peasants. They should look like relatives.

In this example brothers and sisters indeed corresponds to the set of brother-and-sister tuples. To sum up, my account and the possibility of double star application correctly derive the range of possible interpretations for conjoined plural RNs.

\footnotetext{
${ }^{10}$ The importance of the examples like (22) was first pointed out to me by Sergei Tatevosov.
} 


\section{Lexical Restrictions on Reciprocal Plurality}

It seems quite clear, that not all the (pairs of) RNs allow for reciprocal plurality in coordination construction and in plural. In this Section, I will provide a unified account of lexical restrictions on reciprocal plurality and show how it bears on the previous work on the theta-roles of RNs (Barker and Dowty 1993).

As I mentioned in Section 2, many of the pairs of RNs giving rise to reciprocal conjunction have disjoint denotations. For instance, this is true for denotations of brother and sister, teacher and pupil, employer and employee etc. This might be thought of as a preliminary generalization, but the picture turns out to be more complicated. As for the restrictions on reciprocal plurals, the most prominent candidate so far is Eschenbach's assumption that a RN should denote a non-antisymmetric relation to give rise to reciprocal plural interpretation.

However, some interesting cases of kinship ${ }^{11}$ raise a serious challenge to both generalizations above. Assume for instance that in model M, a nephew of John (named Harry in the figure below) marries his aunt. Then he will be both John's uncle and John's nephew.

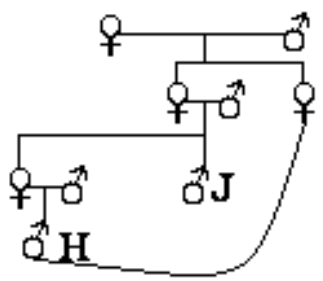

Figure 1: Harry is both an uncle and a nephew of John

The same is of course true for John in this model. Uncle' and nephew' do not have an empty intersection in M. Both John and Harry belong to the denotations of both nouns in this model. However, as illustrated by my example (8), uncle and nephew clearly can have a reciprocal meaning.

Similarly, this model proves, that uncle' $(x)(y)$ does not imply $\neg u_{n c l e}(y)(x)$ and hence the denotation of uncle is non-antisymmetric. But uncles cannot mean 'uncles of each other' and therefore this is also a counterexample to Eschenbach's generalization about reciprocal plurals.

We clearly need an alternative analysis of lexical restrictions on reciprocal plurality. Intuitively, to give rise to reciprocal plurality two RNs should denote inverse relations. This is true for instance for uncle and nephew as uncle' $(x)(y)$ entails nephew' $(y)(x)$. More generally, I will call the two relations inverse if $\mathrm{R}_{1}(\mathrm{x})(\mathrm{y})$ implies $\mathrm{R}_{2}(\mathrm{y})(\mathrm{x})$.

This seems to be too strong as a restriction on reciprocal plurality, as for instance $\operatorname{brother}^{\prime}(x)(y)$ does not imply $\operatorname{sister}^{\prime}(y)(x)$ but brother and sister can be reciprocal. I follow Schwarz (2006) in developing the idea of Strawsonentailment (Von Fintel 1999) as a way of loosening the entailment requirement.

\footnotetext{
${ }^{11}$ I am grateful to Eytan Zweig for pointing out the situations of this kind to me.
} 
Von Fintel (1999) argues that Strawson-entailment is relevant for NPIlicensing. The formal definition of Strawson-entailment is given in (23).

A Strawson-entails B iff the conjunction of A and the presupposition of B entails B. I will write this as $A \stackrel{s}{\rightarrow} B$.

Clearly, if A entails B, A always Strawson-entails B.

Schwarz (2006) demonstrates that gender information in sister ${ }^{\prime}$ is presuppositional as the examples in (24) all convey that Kim is female.
a. Kim isn't his sister
b. Perhaps Kim is his sister
c. Is Kim his sister?

Hence the conjunction of $\operatorname{brother}^{\prime}(x)(y)$ and the presupposition of $\operatorname{sister}^{\prime}(y)(x)$ clearly entails $\operatorname{sister}^{\prime}(y)(x)$. This allows us to adopt Strawsoninverseness as a general restriction on reciprocal plurality.

(25) Two (possibly identical) relations $\mathrm{R}_{1}$ and $\mathrm{R}_{2}$ allow for reciprocal plurality iff they are Strawson-inverse, that is if $R_{1}(x)(y) \stackrel{s}{\rightarrow} R_{2}(y)(x)$.

This restriction gives the right result for the case of model $\mathrm{M}$ depicted in Figure 1. Although uncle' and nephew' are not disjoint, uncle' $(x)(y)$ entails (and hence Strawson-entails) nephew' $(y)(x)$. Therefore, the reciprocal conjunction is well-formed. In the case of plurals, we substitute the same relation $\mathrm{R}$ for both relation variables in (25) and get Strawson-symmetry first proposed by Schwarz (2006). Thus, uncles cannot be reciprocal because uncle' $(x)(y)$ doest not Strawson-entail uncle' $(y)(x)$.

Let us now consider the connection between the proposed lexical restrictions on reciprocal plurality and our derivation. It turns out that the pairs of relations that take part in the derivation of reciprocal plurality always have Strawson-inverse denotations. Somewhat loosely, we may say that the only difference between those relations can be avoided by applying inv to one of them.

$I n v$ can be viewed as an operator that adjusts two RNs to make the relation they have in common explicit or to abstract away from particular order of arguments and indicate that the relation should hold in both directions. The whole derivation of reciprocal plurality is triggered by the fact that the relations in question are nearly inverse. In other words, the lexical restrictions on reciprocal interpretation motivate $i n v$ as a special kind of adjustment operator.

It is also worth noticing that Strawson-inverseness is predictable on the basis of lexical properties of RNs that have been argued to be relevant for the realization of their arguments in possessive construction. Barker and Dowty (1993) suggest that the properties of nominal arguments that are responsible for their realization as either referents or correlates of RNs can be formulated in terms of nominal proto-roles. The nominal proto-roles they propose are proto-part and proto-whole. The properties of proto-part include being located at the boundary 
of other relatum and being a property of it. Proto-whole usually entirely contains the other relatum and is a concrete entity. The nominal argument that is closer to proto-part is predicted to be realized as a referent and the argument that is closer to proto-whole is predicted to be realized as a correlate.

Barker and Dowty claim that if two places of a relation $\mathrm{R}$ are asymmetric in terms of proto-roles this relation is likely to be lexicalized as one RN. However, if none of the arguments of a relation has more proto-part/proto-whole properties; such a relation is predicted to be lexicalized as two RNs that differ only in the order of arguments.

For instance, as the two siblings are not asymmetric in terms of protoroles, the siblinghood relation is predicted to be realized by two RNs denoting the inverse relations. Barker and Dowty assume that the nouns brother and sister confirm this prediction. They notice that the two nouns are not, strictly speaking, inverse but claim that they are nearly inverse.

My findings can easily be correlated with the findings of Barker and Dowty. First, the notion of Strawson-inverseness can help to make their claim about nearly inverse nouns more precise. In fact what they mean by nearly inverse turns out to be equivalent to my notion of Strawson-inverseness. This includes the RNs with inverse denotations like uncle and nephew but also extends to the pairs like brother and sister, father and son etc. Second, my analysis gains additional support from this observation. The lexical properties of RNs responsible for argument realization seem to predict which pairs of nouns will be Strawsoninverse. Hence, Strawson-inverseness is not a lexical property of RNs stored in the dictionary just to license reciprocal plurality. Rather this property and the restrictions on reciprocal plurality can be derived from independently needed lexical information.

Finally, I would like to consider the implications of the observations presented in this section for what I said about the status of reciprocal conjunction with respect to intersective and group-forming conjunction. In Section 2, I argued that reciprocal conjunction is nearly in complementary distribution with the intersective conjunction. However, the situation, depicted in Figure 1 apparently licenses both types of interpretations.

John invited an uncle and nephew to the party

In other words, the example (8) repeated here as (26) can in fact be 3-ways ambiguous between reciprocal, group-forming and intersective conjunction. However, the intersective interpretation in (26) and the whole situation in Figure 1 are clearly marginal. I do not think such cases undermine my claim that the reciprocal conjunction is a variant of intersective conjunction. It is just that in some rare cases both variants can apply.

\section{Residual Issues and Possible Extensions of my Analysis}

In my treatment of conjoined and plural RNs, I have mainly focused on the semantics and put aside the pragmatic and syntactic aspects of the analysis. 
However, as I noted in Footnote 4 the analysis of examples like $a$ husband and $a$ wife might appeal to pragmatic principles governing coordination.

Similarly, I would attribute to pragmatic reciprocity the quasi-reciprocal conjunctions of three kinship terms like husband, wife and mother-in-law ${ }^{12}$. Several peculiarities of such examples indicate that the RNs in such cases have probably shifted to 1-place role predicates and that reciprocity is purely pragmatic. To begin with, the relation is not reciprocal with respect to all three conjuncts. Rather than reciprocity, the role labels mentioned are important. Thus, husband, wife and mother cannot have a meaning synonymous to that of husband, wife and mother-in-law though the referent in question is mother to one of the conjoined referents and mother-in-law to the other one ${ }^{13}$.

In addition to such pragmatic mechanisms, the extension of my analysis to covert reciprocity in other categories also deserves further investigation. Krifka (1991) proposes an analysis of covertly reciprocal verbs like meet similar to Eschenbach's (1993) analysis of plural RNs (cf. also the recent attempt by Rubinstein 2006 where she assumes the complex verbs with possible changes in the order of arguments). In addition to those, the possessive morphemes in some languages are also used to express reciprocal plurality. The study of reciprocity in other categories may make the syntactic status of inv clearer.

\section{Conclusion}

I have analyzed the reciprocal interpretation of RNs in coordinate structures and in plural. I have argued that the similarity in the two interpretations is not accidental and proposed an analysis that captures both interpretations in a similar way.

My analysis allows the reciprocal conjunction to be treated as a variant of intersective conjunction and hence does not extend the range of interpretations of and. However, my data on coordination of RNs demonstrate that the unified accounts of coordination are not fully successful: the account of Winter (2001) has to face the examples of split reading and the account of Heycock and Zamparelli (2005) is hard to generalize to RNs.

In the case of reciprocal plurals, my analysis allows to derive both strong and weak readings. It also makes the right predictions for the conjoined plurals ambiguity.

Finally, the application of inv coincides quite well with lexical restrictions on reciprocal plurality. I propose Strawson-inverseness as a key restriction here. I have argued that Strawson-inverseness can be predicted on the basis of independently motivated lexical properties of RNs.

\section{References}

\footnotetext{
${ }^{12}$ Such cases were first pointed out to me by an anonymous SALT reviewer.

${ }^{13}$ Laurence Horn (p.c.) suggests that such contrasts probably have to do with the fact that RNs activate relations, not only referents in discourse.
} 
Asudeh, Ash: 2005, 'Relational Nouns, Pronouns, and Resumption', Linguistics and Philosophy 28:4: 375-446.

Barker, Chris: 1999, 'Temporary Accommodation: I am the Oldest of My Siblings', Studies in the Linguistic Sciences vol. 29:1: 3-13.

Barker, Chris and David Dowty: 1993, 'Non-verbal Thematic Proto-roles', in: Amy Schafer (ed.) Proceedings of NELS 23: 49-62, GSLA, Amherst.

Beck, Sigrid: 2000, 'Star Operators Episode 1: Defense of Double Star', in Kiyomi Kusumoto and Elisabeth Villalta (eds.) University of Massachusets Occasional Papers in Linguistics 23. Issues in Semantics: 1-23, GLSA, University of Massachusets, Amherst.

De Bruin, Jos and Remko Scha: 1988, 'The Interpretation of Relational Nouns', in Steven Bird (ed.) Meeting of the Association for Computational Linguistics: 25-32, ACL, Morristown, NJ.

Eschenbach, Carola: 1993, 'Semantics of Number', Journal of Semantics 10: 131.

Hackl, Martin: 2002, 'The Ingredients of Essentially Plural Predicates', in Masako Hirotani (ed.) Proceedings of the 32nd North East Linguistic Society conference: 171-182, GLSA, University of Massachusetts, Amherst.

Heycock, Caroline and Roberto Zamparelli: 2003, 'Coordinated Bare Definites', Linguistic Inquiry 34:3: 443-469.

Heycock, Caroline and Roberto Zamparelli: 2005, 'Friends and Colleagues: Plurality, Coordination, and the Structure of DP', Natural language semantics 17:3: 201-270.

Krifka, Manfred: 1986, Nominalreferenz und Zeitkonstitution: Zur Semnatik von Massentermen, Pluraltermen und Aspektklassen. Doctoral dissertation, University of Munich.

Krifka, Manfred: 1990, 'Boolean and Non-boolean and', in: L. Kálman and L. Polos (eds.) Papers from the Second Symposium on Logic and Language: 161-188, Budapest, Akadémiai Kiadó.

Krifka, Manfred: 1991, 'How to Get rid of Groups Using DRT', in Texas linguistics forum 32: discourse: 71-109.

Lander, Yury: 2000, 'K tipologii reljacionnyh imen' [On the Typology of Relational Nouns, in Russian]. Ms. Russian State University for Humanities.

Link, Godehard: 1983, 'The Logical Analysis of Plurals and Mass Terms: a Lattice-theoretical Approach', in: R.Bauerle et al. (eds.) Meaning, Use, and Interpretation of Language: 303-323, De Gruyter, Berlin and New York.

Partee, Barbara H.: 1989, 'Binding Implicit Variables in Quantified Contexts' in: C. Wiltshire, B. Music and R. Graczyk (eds.) CLS 25: Papers from the Twenty Fifth Meeting of the Chicago Linguistic Society: 342-365.

Partee, Barbara H.: 1999, 'Weak NP's in HAVE Sentences', in J. Gerbrandy et al. (eds.), JFAK [a Liber Amicorum for Johan van Benthem on the occasion of his 50th Birthday], CD-Rom, Amsterdam, University of Amsterdam. 
Partee, Barbara H. and Mats Rooth: 1983, 'Generalized Conjunction and Type Ambiguity', in: R. Bauerle et al. (eds.) Meaning, Use and Interpretation of Language: 361-383, De Gruyter, Berlin and New York.

Roodenburg, Jasper: 2004, 'French Bare Arguments are not Extinct', Linguistic Inquiry 35:2: 301-313.

Schwarz, Bernard: 2006, 'Covert Reciprocity and Strawson-symmetry', Snippets 13.

Schwarzschild, Roger: 1992, 'Types of Plural Individuals', Linguistics and philosophy 15:6: 641-675.

Vikner, Carl and Per A. Jensen: 2002, 'A Semantic Analysis of the English Genitive. Interaction of Lexical and Formal Semantics', Studia Linguistica 56: 191226.

Von Fintel, Kai: 1999, 'NPI-licensing, Strawson-Entailment, and Context Dependency', Journal of semantics 16: 97-148.

Winter, Yoad: 2001, Flexibility Principles in Boolean Semantics: the Interpretation of Coordination, Plurality and Scope in Natural Language, Current Studies in Linguistics, 37, Cambridge, MA, MIT Press. 